\title{
Desenvolvimento e evidências de validade de uma escala de autoeficácia para situações de vitimização
}

\author{
Daniela Cristina Campos \\ Margareth Regina Gomes Veríssimo de Faria \\ Daniela Sacramento Zanini \\ Pontificia Universidade Católica de Goiás, GO, Brasil \\ Evandro Morais Peixoto \\ Pontifícia Universidade Católica de Campinas, SP, Brasil
}

\begin{abstract}
Resumo
Vitimização é a experiência de violência. Ela pode ocasionar sequelas físicas e psicológicas e aumentar a vulnerabilidade do indivíduo sofrer outras vitimizações. A autoeficácia é a crença que o indivíduo tem sobre sua capacidade de produzir os resultados desejados em determinada situação. O objetivo deste estudo foi elaborar e investigar as primeiras evidências de validade e precisão da Escala de Autoeficácia Relacionada à Experiências de Vitimização, em uma amostra de 210 estudantes universitários. A escala foi elaborada com 17 itens, retidos após análise de conteúdo. Análise Fatorial Exploratória e Análise Paralela indicaram a pertinência da solução unifatorial (15 itens), com boa precisão $\alpha=0,88$. Através do Modelos de Créditos Parciais descreveu-se os parâmetros dos itens e características dos participantes (dificuldade/theta, índices de ajustes infit/outfit), assim como a invariância dos parâmetros dos itens em função do sexo dos participantes, o que demonstrou adequação do instrumento para avaliação do construto em questão.

Palavras-chave: Autoeficácia; Vitimização; Escala; Percepção social; Teoria de resposta ao item.
\end{abstract}

\section{Development and validity evidence of a self-efficacy scale for victimization situations}

\begin{abstract}
Victimization is the experience of violence. It may lead to physical and psychological sequelae and increase the vulnerability that the individual suffers another victimization. Self efficacy is the belief that the individual has on his ability to produce the desired results in a given situation. This study aimed to develop and estimate the first reliability and validity evidences of the Self-efficacy Scale Related to Victimization Experiences, in a sample composed by 210 undergraduate students. The scale was developed with 17 items, maintained after content analysis. The Exploratory Factor Analysis and Parallel Analysis indicated the relevance of the one-factor solution (15 items), with good reliability $\alpha=0.88$. Through Rasch-Masters Partial Credit Mode it was described the item parameters and participants' characteristics (difficulty levels/theta and adjustment indices infit/outfit), as well as the item parameters invariance according to the participants gender, which demonstrated the instrument suitability for evaluation of the construct in the matter.
\end{abstract}

Keywords: Self-efficacy; Victimization; Scale; Social perception; Item response theory.

\section{Desarrollo y evidencias de la validez de una escala de auto-eficacia para situaciones de victimización}

\section{Resumen}

Victimización es la experiencia de violencia. Ella puede conducir a consecuencias físicas y psicológicas y aumentar la vulnerabilidad de la persona a otra victimización. La autoeficacia es la creencia que el individuo tiene sobre su capacidad para producir los resultados deseados en una situación. El objetivo de este estudio fue desarrollar e investigar la primera evidencia de la validez y fiabilidad de la Escala de Autoeficacia relacionada a las experiencias de victimización en una muestra de 210 estudiantes universitarios. La escala fue elaborada con 17 ítems, retenidos después de análisis de contenido. Análisis factorial exploratorio y Revisión paralela indican la pertinencia de la solución de un factor ( 15 artículos), con buena precisión $\alpha=0,88$. Por medio de los Modelos de Créditos Parciales se describió los parámetros de los ítems y las características de los participantes (dificultad/theta, índice de ajuste infit/outfit) y la invariancia de los parámetros de los ítems según sexo de los participantes, lo que demuestra la adecuación del instrumento para evaluar el constructo en cuestión.

Palabras-clave: Autoeficacia; Victimización; Escala; Percepción social; Teoría de respuesta al ítem. 


\section{Introdução}

De acordo com o Ministério da Saúde, a violência é um fenômeno social que atinge toda a população mundial podendo fazer vítimas em momentos específicos da vida ou de forma reiterada. Ela pode ser perpetrada por indivíduos, grupos e nações, ocasionando danos físicos, emocionais e psicológicos ao próprio indivíduo e a terceiros (Brasil, 2009). Sobre as consequências da violência, Dahlberg e Krug (2007) apontam que ela pode gerar vítimas fatais e outras que não chegam à morte, porém, sofrem lesões resultantes de autoagressão, violência perpetrada por um terceiro ou violência coletiva. Apesar disso, algumas variáveis parecem influenciar não só a vivência de violência, mas também as consequências desta vivência sobre a saúde dos indivíduos. Neste sentido, a crença de um indivíduo sobre sua capacidade de enfrentar episódios de violência (crença de autoeficácia aplicada à violência) poderia ser uma destas variáveis. Assim, o presente artigo pretende desenvolver uma escala de autoeficácia para situações de vitimização, estimar suas primeiras evidências de validade com base na estrutura interna e precisão, descrever as propriedades dos itens e características dos participantes da pesquisa.

O Sistema de Informações de Mortalidade (SIM) apontou que entre os anos 1980 e 2012 o quantitativo de mortes totalizou 2.459.791 vítimas. Destas, 1.202.245 eram vítimas de homicídio; 1.041 .335 de acidentes de transporte e 216.211 por suicídio (Waiselfisz, 2014). Estima-se que a violência seja a principal causa das mortes da população entre um e 39 anos, sendo considerada a terceira maior causa da morte da população geral (Brasil, 2009).

$\mathrm{Na}$ atualidade, as mais diversas formas de violência podem se expressar cotidianamente, como a violência urbana, doméstica, sexual, social, no trabalho, entre outras (Azevedo, 2013). Essas violências podem se manifestar por ações de diversas naturezas, como física, sexual, psicológica e negligência ou privação (Brasil, 2009).

A violência física caracteriza-se pela tentativa de causar dano não acidental a uma pessoa por meio de força física ou arma, podendo provocar lesões internas, externas ou ambas. A violência sexual é toda situação na qual uma pessoa obriga a outra a executar ato sexual contra sua vontade, por meio de coerção física ou intimidação psicológica. A violência psicológica pode manifestar-se por ação ou omissão, que tenha como objetivo depreciar a autoestima e a identidade da pessoa por meio de agressões verbais e/ou humilhações constantes. Finalmente, a negligência ou privação se caracteriza pela ausência de atendimento às necessidades básicas físicas e emocionais das pessoas (Brasil, 2009).

A experiência de violência vivenciada pela vítima é chamada vitimização e, por essa nomeação, as pessoas podem dar um significado ao ocorrido, assim como partilhar suas experiências com outras pessoas que também passaram por situações semelhantes (Azevedo, 2013). Para Finkelhor (2007), a vitimização é definida como os danos causados às pessoas por ações de outros indivíduos, que se comportam de maneira a violar as normas sociais, possuindo um potencial especial para causar um impacto traumático.

Corroborando a afirmação de Finkelhor (2007) sobre o impacto traumático das situações de violência, uma pesquisa realizada por Williams, D'Affonseca, Correia e Albuquerque (2011) buscou investigar experiências negativas vivenciadas por uma amostra de estudantes universitários nos anos escolares anteriores à universidade. Os resultados desse estudo indicaram que as situações de violência vividas pelos participantes eram, em sua maioria, de natureza verbal $(45,3 \%)$, sendo o agressor outro estudante (52\%). Dos participantes vitimizados, $89 \%$ relataram que, após a experiência de violência, apresentaram sintomas como nervosismo, raiva, tristeza, solidão e rememoração do fato. Destes, 38\% demonstraram quadro sugestivo de transtorno de estresse pós-traumático.

Outro estudo que também corrobora a afirmação de Finkelhor (2007) foi o realizado por Martins (2011), que teve como objetivo investigar a percepção de mulheres vítimas de abusos sexuais sobre os impactos gerados pela situação de violência em termos físicos, psicológicos e comportamentais e as estratégias utilizadas para lidar com esses impactos. Os resultados revelaram que todas as participantes narraram sintomas como isolamento, medo generalizado, depressão, baixa autoestima e indicativos de estresse pós-traumático.

No entanto, esses resultados mostraram também que as mulheres que sofreram violência sexual não reagiram da mesma forma à situação vivida. Como estratégias de enfrentamento foram usadas a busca de apoio de pessoas próximas e os serviços voltados para o atendimento de vítimas de violência. Aquelas que puderam contar com o apoio de pessoas próximas relataram ter superado ou amenizado os sintomas de forma mais rápida e mostrando mais resiliência frente à situação do que aquelas que não tiveram esse apoio (Martins, 2011).

Desse modo, é possível observar que as vivências de situações de vitimização podem ocasionar sequelas físicas e psicológicas em quem as sofre, além da possibilidade de aumentar a vulnerabilidade do indivíduo para que sofra outras situações de vitimização 
ao longo da vida, agravando, portanto, seu nível de sofrimento e suas possibilidades de adoecimento. Contudo, alguns fatores parecem interferir nesta relação entre vitimização e adoecimento. Entre eles pode-se citar o apoio social, a rede de proteção social, o tipo de vitimização, a idade, o sexo, a autoeficácia percebida, entre outros (Martins, 2011; Waiselfisz, 2014; Williams et al., 2011).

O conceito de autoeficácia surgiu com Bandura, em 1977 e se refere à crença que o indivíduo tem sobre sua capacidade de produzir os resultados desejados em determinada situação assim como superar dificuldades, apresentando controle sobre o ambiente (Bandura, 1999, 2006). Como a própria definição aponta, trata-se da percepção do indivíduo sobre seu desempenho, e não do desempenho em si. Por essa razão, é comum o uso dos termos autoeficácia percebida ou crenças de autoeficácia (Pajares, 2004, 2005). Uma vez que a autoeficácia pessoal não está diretamente relacionada à capacidade real e objetiva de desempenho, as características físicas, psicológicas ou pessoais dos indivíduos não têm influência direta sobre essas crenças (Zimmerman, 2000).

Duas funções diferentes estão associadas ao processamento cognitivo das crenças de autoeficácia: a primeira se refere ao tipo de informação e ao emprego de indicadores de eficácia pessoal; a segunda envolve a combinação de regras usadas para a integração das informações. As pessoas, por exemplo, fazem julgamentos acerca da sua eficácia com base em suas realizações e desempenho, que podem variar dependendo da interpretação feita, dos preconceitos pessoais, da avaliação da dificuldade da tarefa, se recebeu algum tipo de auxílio, das condições nas quais as tarefas foram realizadas, do estado emocional e físico no momento, da sua evolução ao longo do tempo, além da lembrança de suas realizações. Esses fatores compõem a primeira função utilizada (Bandura, 1999).

A segunda função se estrutura com base nas quatro fontes de influência das crenças de autoeficácia: experiências pessoais, experiências vicárias, persuasão verbal e aspectos fisiológicos (Bandura, 1999). As experiências pessoais registram o aprendizado da pessoa de acordo com suas vivências e as vicárias o aprendizado adquirido com as experiências de outras pessoas (Bandura, 1999). A persuasão verbal se se refere ao quanto as pessoas que compõem a rede de relações dos indivíduos estão convencidas de que eles dispõem do necessário para alcançar sucesso, expressando essas crenças por meio de feedbacks (Bandura, 1999). A quarta fonte diz respeito ao equilíbrio emocional e a suas manifestações fisiológicas percebidas pelo indivíduo (Bandura, 1999; Salvetti, Pimenta, Lage, Oliveira Júnior, \& Rocha, 2007).
Para Bandura (2006), as crenças de autoeficácia não correspondem a um traço global, e sim a um conjunto de percepções vinculadas a experiências específicas. De acordo com Zimmerman (2000), além dessas experiências, o indivíduo também estrutura suas crenças com base no contexto em que está inserido. Dessa forma, mostra-se mais adequada a construção de escalas de autoeficácia percebida voltada para o domínio do objeto que se pretende investigar em seu contexto específico (Bandura, 2006).

No levantamento bibliográfico realizado na literatura internacional e nacional foram encontradas algumas escalas de autoeficácia em variados contextos. No contexto brasileiro foram encontradas pesquisas como a de Finotelli Junior e Capitão (2011) que desenvolveram um estudo com o objetivo de levantar evidências de validade da versão brasileira da Escala de Autoeficácia Sexual - Função Erétil (SSES-E). Ambiel e Noronha (2011) elaboraram uma Escala de Autoeficácia para Escolha Profissional (EAE-EP), embora não tenham apontado índices estatísticos de validade e confiabilidade. Oliveira, Silva, Garcia, Melo-Silva e Teixeira (2014) desenvolveram um estudo cujo objetivo foi adaptar e verificar as evidências de validade para a escala de autoeficácia no aconselhamento de carreira para brasileiros. Polydoro e Casanova (2015) buscaram adaptação e a busca de evidências psicométricas de uma escala de autoeficácia acadêmica para estudantes do ensino médio. Mognon e Santos (2016) construíram uma Escala de Autoeficácia para dirigir e buscaram identificar as propriedades psicométricas da mesma.

Observa-se que embora elaboradas para contextos específicos estas escalas não compreendem as situações de vitimização. Desse modo, faz-se necessário a disponibilidade de um instrumento que avalie a crença de autoeficácia aplicada a situações de vitimização e que conte com evidências de validade. A fim de contribuir para o preenchimento desta lacuna, esta pesquisa teve como objetivos desenvolver uma escala de autoeficácia para situações de vitimização, estimar suas primeiras evidências de validade com base na estrutura interna e precisão, descrever as propriedades dos itens e características dos participantes da pesquisa.

\section{Método}

\section{Participantes}

Participaram desta pesquisa 210 estudantes universitários com idade ente 18 e 59 anos $(25,23 \pm 7,14$, $80,6 \%$ mulheres). Destes, $66,7 \%$ eram solteiros, $28,6 \%$ casados, 3,8\% divorciados e 6,5\% não informaram o estado civil. Com relação ao número de irmãos, $8,1 \%$ eram filhos únicos, 65,1\% tinham de 1 a 3 irmãos, 
$16,2 \%$ de 4 a 6 irmãos, 5,2\% de 7 a 10 irmãos e 3,8\% tinham acima de 10 irmãos. Dos participantes do estudo, 32,4\% cursavam Psicologia, 25,7\% Gestão de Recursos Humanos, 22,6\% Fisioterapia, 16,2\% Administração, 0,5\% Educação Física e 0,5\% Enfermagem. Quanto a origem das instituições, tratavam-se de duas IES privadas localizadas na região Centro-Oeste do Brasil.

\section{Instrumento}

Para esta pesquisa, foi desenvolvida uma escala de avaliação de autoeficácia relacionada a experiências de vitimização. Originalmente, a escala foi construída com 17 itens, com base na leitura do referencial teórico do tema vitimização, assim como de outras escalas de autoeficácia geral e específicas para outros contextos (Ambiel \& Noronha, 2011; Guerreiro \& Polydoro, 2010; Finotelli Junior \& Capitão, 2011; Martins \& Ribeiro, 2008; Oliveira et al., 2014). Itens que deveriam ser respondidos seguindo a instrução: "Por favor, responda às perguntas abaixo dizendo o quanto você concorda com cada afirmação, relativo ao que você pensa sobre você mesmo". Os participantes deveriam responder assinalando uma das cinco respostas possíveis para cada questão, utilizando uma escala Likert de cinco pontos, que variava de 1 (discordo totalmente) a 5 (concordo totalmente).

O conjunto de itens foi submetido à avaliação de cinco juízes, previamente treinados, para verificação da objetividade e pertinência dos conteúdos expressos pelos itens e abrangência do conjunto de itens em relação com o construto que se pretendia medir. Após as análises individuais, foram calculados acordos entre as respostas dos juízes, e retidos os itens que apresentavam acordo, obtido com o cálculo Kappa superiores a 0,75 (Fonseca, Silva, \& Silva, 2007). Dessa forma, todos os 17 itens foram retidos, dando origem a primeira versão da Escala de Autoeficácia Relacionada à Experiências de Vitimização (EAREV).

Dados sociodemográficos: esta ferramenta teve como principal objetivo a investigação de características da amostra como idade, sexo, estado civil, número de irmãos e curso universitário.

\section{Procedimentos}

Coleta de dados. A coleta de dados foi realizada após aprovação do projeto pelo Comitê de Ética sob o Protocolo 0113.0.168.000-06 e consentimento formal dos participantes através da assinatura do Termo de Consentimento Livre e Esclarecido (TCLE). A aplicação ocorreu de forma coletiva, nas próprias salas de aula dos alunos, em seus respectivos cursos, por aplicadores previamente treinados, nos meses de novembro e dezembro de 2014.
Análise de dados: para estimativa da estrutura interna da Escala empregou-se Análise Fatorial Exploratória (AFE) com método de estimação Unweighted Least Squares (ULS) e método de rotacional oblíquo Promax no software estatístico Factor versão 9.3 (Lorenzo-Seva, Timmerman \& Kiers, 2011). Considerando o tipo de medida, variáveis ordinais, e violação do pressuposto de normalidade multivariada (Mardia $=20,411, p \leq 0,01$; Mardia, 1970) as análises foram baseadas em uma matriz de correlação policórica. Para a retenção do número de fatores recorreu-se a diferentes critérios descritos na literatura: Critério Guttman-Kaiser, Análise Paralela (AP) baseada em Minimum Rank Factor Analysis (ver Timmerman \& Lorenzo-Seva, 2011), cargas fatoriais superiores a 0,32, e interpretabilidade da solução fatorial. Para análise da consistência interna empregouse o coeficiente alfa de Cronbach, para qual índices superiores 0,7 são esperados (Tabachnick \& fidell, 2012).

Para estimação das propriedades dos itens e das características dos participantes recorreu-se a Teoria de Resposta ao Item (TRI), Rasch-Master's Partial Credit Model (Modelo de Créditos Parciais) (Wright, \& Masters, 1982), estimado a partir do método Joint Maximum Likelihood no software WINSTEPS 3.7 (Linacre, 2015). Modelo matemático que permite estimar, de forma independente, os parâmetros de dificuldade dos itens $(\delta)$ e o nível de traço latente das pessoas (theta, $\theta$ ) através de resposta à itens politómicos (Amantes \& Coelho, 2015). A relação entre os parâmetros dos itens, características das pessoas e a probabilidade de resposta correta ao item é expressa pela Curva Características do Item (CCI). Desta forma, o modelo matemático pressupõe que, quanto maior a intensidade do traço latente para pessoa, frente ao conteúdo investigado pelo item, maior será a probabilidade de que ela concorde com o item, por outro lado, quanto menos intensa forem as características descritas pelos itens para a pessoa, menor será a probabilidade de que ela concorde com o mesmo.

Com base nesta proposta, foram estimados os parâmetros dos itens, dificuldade (parâmetro $b$ ), características das pessoas (theta $\theta$ ) e índices de ajuste (Infit/Outifit) que devem se situar entre 0,5 e 1,5 (Linacre, 2015). Além disso, buscou-se estimar a invariância dos parâmetros dos itens em função do sexo dos participantes. Para tanto recorreu-se ao Differential Item Functioning (DIF), Funcionamento Diferencial dos Itens, que descreve a diferenças na probabilidade de endosso ao item entre pessoas com mesmo nível de traço latente. Foram adotados como 
critérios para presença de DIF valores de contraste superiores a 0,42 ou probabilidade menor que 0,05 , conforme recomendado por Aguerri, Galibert, Lozzia, Abal, e Attoresi (2007).

\section{Resultados e Discussão}

\section{Estrutura interna e precisão}

AAFE foi antecedida da verificação dos indicadores de adequação da matriz de correlação, estatística KMO igual a 0.896 e teste de Esfericidade de Bartlett igual a $\chi^{2}(105)=1173,7 \mathrm{p}<0.01$, valores que indicam a pertinência dos cálculos fatoriais. Avançou-se, então, para AFE e avaliação dos critérios retenção de fatores: Critério Guttman-Kaiser e AP, que indicaram a pertinência de solução unifatorial, visto que apenas o primeiro eigenvalue apresentou índice superior a um $(5,270)$ seguido do segundo e terceiro $(0.958$ e 0.543 , respectivamente). A partir da AP foram geradas aleatoriamente 500 matrizes de correlação policóricas pelo método Permutation of theraw data (Buja \& Eyuboglu, 1992). Verificou-se que apenas o primeiro fator obtido através dos dados reais, apresentou índice de variância explicada superior aos fatores obtidos por meio dos dados aleatórios, mais especificamente aqueles alocados no percentil 95 (Lorenzo-Seva \& Ferrando, 2006). São elas 40,8\%, 11,6\% e 8,2\%, para os três primeiros fatores extraídos dos dados reais contra $13,4 \%, 12,1 \%$ e $11,1 \%$, dos três primeiros fatores extraídos dos dados aleatórios. A solução unifatorial é apresentada na Tabela 1, na qual se observa as comunalidades e cargas fatoriais apresentadas por cada item, porcentagem de variância explicada e índices de consistência interna, alfa de Cronbach do fator.

Observa-se na Tabela 1 que com exceção dos itens 16 e 17 que não apresentaram cargas fatoriais iguais ou superiores a 0,32 , todos os itens se apresentaram como bons representantes do construto medido pelo fator, uma vez apresentaram comunalidades iguais ou superiores a 0,393 e cargas fatorais iguais ou superiores a 0,402. Quanto a porcentagem de variância explicada verifica-se que a solução explica cerca de $40,8 \%$ da variância total, resultados que demostram a pertinência da solução unifatorial com 15 itens. Em relação a interpretação teórica do fator, notadamente o conjunto de itens avalia a crença ou percepção dos sujeitos quanto sua capacidade de fazer frente a situações de violência, seja através de recursos próprios ou através do apoio de outras pessoas. E, portanto, foi denominado crença de eficácia geral frente a situações de vitimização.

Uma vez estabelecida estrutura interna da EAREV-15 buscou-se estimar as primeiras evidências de precisão da escala através do cálculo do coeficiente alpha de Cronbach. Observou-se índice de consistência interna igual 0,88 (ver Tabela 1 ), resultado que pode ser interpretado como bom indicador de precisão da escala (Tabachnick \& Fidell, 2012).

TABELA 1

Modelo fatorial da EAREV-17

\begin{tabular}{|c|c|c|}
\hline Itens da escala & $h$ & $h^{2}$ \\
\hline $\begin{array}{l}1 \text { - Sempre que me deparo com uma situação } \\
\text { de violência, sinto que posso resolvê-la. }\end{array}$ & 0.594 & 0.530 \\
\hline $\begin{array}{l}2 \text { - Se eu me esforçar o suficiente, posso } \\
\text { resolver a situação de violência. }\end{array}$ & 0.738 & 0.532 \\
\hline $\begin{array}{l}3 \text { - Se eu procurar ajuda, posso resolver a } \\
\text { situação de violência. }\end{array}$ & 0.479 & 0.402 \\
\hline $\begin{array}{l}4 \text { - Se alguém não me apoia quando busco } \\
\text { ajuda para uma situação de violência, eu } \\
\text { mesmo encontro maneiras de me proteger. }\end{array}$ & 0.393 & 0.439 \\
\hline $\begin{array}{l}5 \text { - Eu confio nas minhas habilidades para } \\
\text { resolver situações violentas. }\end{array}$ & 0.682 & 0.678 \\
\hline $\begin{array}{l}6 \text { - Estou confiante de que eu poderia lidar } \\
\text { bem com acontecimentos inesperados de } \\
\text { violência. }\end{array}$ & 0.724 & 0.665 \\
\hline $\begin{array}{l}7 \text { - Graças a minha habilidade, eu posso me } \\
\text { proteger de situações de violência. }\end{array}$ & 0.619 & 0.629 \\
\hline $\begin{array}{l}8 \text { - Sei que posso resolver a maioria das } \\
\text { situações que envolvem violência no meu } \\
\text { contexto familiar e social. }\end{array}$ & 0.543 & 0.625 \\
\hline $\begin{array}{l}9 \text { - Eu posso permanecer calmo quando } \\
\text { enfrento situações de violência, porque } \\
\text { confio na minha habilidade para enfrentar } \\
\text { a situação. }\end{array}$ & 0.649 & 0.636 \\
\hline $\begin{array}{l}10 \text { - Quando enfrento um problema } \\
\text { relacionado a violência contra mim ou a } \\
\text { minha família, sei que posso encontrar várias } \\
\text { soluções. }\end{array}$ & 0.747 & 0.547 \\
\hline $\begin{array}{l}11 \text { - Se eu for vítima de algum tipo de } \\
\text { violência, sei que posso encontrar uma forma } \\
\text { de evitar que isso ocorra novamente. }\end{array}$ & 0.619 & 0.473 \\
\hline $\begin{array}{l}12 \text { - Eu posso direcionar os acontecimentos } \\
\text { da minha vida, assim como posso evitar } \\
\text { situações que possam me afetar de forma } \\
\text { violenta. }\end{array}$ & 0.591 & 0.497 \\
\hline $\begin{array}{l}13 \text { - Embora eu não tenha sofrido violência, } \\
\text { eu sei me defender quando me deparo com } \\
\text { situações violentas. }\end{array}$ & 0.502 & 0.558 \\
\hline $\begin{array}{l}14 \text { - Eu sempre consigo sair de situações de } \\
\text { violência. }\end{array}$ & 0.513 & 0.567 \\
\hline $\begin{array}{l}15 \text { - As pessoas acreditam que sou capaz de } \\
\text { lidar com situações de violência. }\end{array}$ & 0.489 & 0.575 \\
\hline $\begin{array}{l}16 \text { - Fico muito nervoso em situações de } \\
\text { violência. }\end{array}$ & $-0,084$ & 0,007 \\
\hline $\begin{array}{l}17 \text { - Perco o controle quando enfrento } \\
\text { situações de violência. }\end{array}$ & $-0,180$ & 0,032 \\
\hline Porcentagem de variância explicada & \multicolumn{2}{|c|}{$40,8 \%$} \\
\hline Alfa de Cronbach & \multicolumn{2}{|c|}{0,88} \\
\hline
\end{tabular}

* Método de extração: ULS (Unweighted Least Squares). 


\section{Propriedades dos itens e características das pessoas}

Tendo obtido as primeiras evidências de validade com base na estrutura interna e precisão da EAREV-15, buscou-se avaliar os parâmetros dos itens e características dos participantes via TRI, mais especificamente a partir Modelo de Créditos Parciais. Assim, estimou-se inicialmente os parâmetros dos itens: dificuldade, erro padrão, índices de ajustes e correlação item-theta, conforme apresentados na Tabela 2.

TABELA 2

Parâmetros dos itens

\begin{tabular}{cccccc}
\hline Item & $b$ & $E P$ & Infit & Outfit & Corr item- $\theta$ \\
\hline 1 & 0,27 & 0,09 & 1,05 & 1,07 & 0,59 \\
2 & $-0,31$ & 0,09 & 1,05 & 1,08 & 0,59 \\
3 & $-1,18$ & 0,09 & 1,30 & 1,29 & 0,44 \\
4 & $-0,51$ & 0,08 & 1,35 & 1,42 & 0,48 \\
5 & 0,15 & 0,09 & 0,84 & 0,86 & 0,68 \\
6 & 0,60 & 0,09 & 0,85 & 0,84 & 0,69 \\
7 & 1,00 & 0,09 & 0,80 & 0,79 & 0,71 \\
8 & 0,28 & 0,09 & 0,89 & 0,88 & 0,67 \\
9 & 0,45 & 0,08 & 0,89 & 0,88 & 0,68 \\
10 & $-0,24$ & 0,09 & 0,97 & 0,99 & 0,61 \\
11 & $-0,83$ & 0,09 & 1,28 & 1,26 & 0,48 \\
12 & $-0,45$ & 0,09 & 1,07 & 1,05 & 0,57 \\
13 & 0,16 & 0,09 & 0,83 & 0,83 & 0,70 \\
14 & 0,45 & 0,10 & 0,90 & 0,90 & 0,65 \\
15 & 0,18 & 0,09 & 0,97 & 0,98 & 0,63 \\
\hline Media & 0 & 0,09 & 1,00 & 1,02 & \\
\hline DP & 0,56 & 0,00 & 0,17 & 0,20 & \\
\hline
\end{tabular}

Destaca-se que no procedimento de ancoragem optou-se por fixar as médias de dificuldade dos itens em zero, desta forma a dificuldade dos itens variaram entre -1,18 (item 3) e 1 (item 7), com desvio padrão igual a 0,56 . Resultados que indicam boa capacidade da escala em cobrir o construto avaliado, aproximadamente 2,18 logit. No entanto, aponta lacunas na avaliação do construto, uma vez que o instrumento não conta com itens muito fáceis ou muito difíceis de serem endossados, imputando maior erro a avaliação de pessoas com níveis de theta fora da faixa avaliada pelos itens. Em relação aos índices de ajuste observa-se que todos os itens se adequaram ao padrão esperado pelo modelo, notadamente os valores infit variaram entre 0,8 e 1,35 e outfit 0,79 e 1,42. Por fim, índices de correlação item-theta variaram entre 0,44 e 0,71 , demostrando índices de correlações moderados e fortes e, portanto, boa capacidade dos itens em recuperar nível de traço latente dos participantes da pesquisa.
Quanto a descrição das características dos participantes, na Tabela 3 são apresentadas estatísticas descritivas média, desvio padrão, mínimo e máximo referente ao traço latente dos participantes, erro padrão e índices de ajustes. Notadamente o valor médio do nível de traço latente dos participantes da pesquisa $(-0,22)$ indica que no geral os itens foram relativamente difíceis de serem endossados. Contudo, as estatísticas mínimo e máximo apontam que a amostra foi composta por sujeitos com diferentes níveis no traço latente, entre $-3,42$ e 4,67.

TABELA 3

Características dos participantes

\begin{tabular}{ccccc}
\hline & $\theta$ & $E P$ & Infit & Outfit \\
\hline Média & $-0,22$ & 0,34 & 1,02 & 1,02 \\
DP & 1,05 & 0,04 & 0,68 & 0,71 \\
Mínimo & 4,67 & 0,76 & 4,71 & 5,27 \\
Máximo & $-3,42$ & 0,31 & 0,15 & 0,15 \\
\hline
\end{tabular}

Quanto aos índices de ajustes o valor médio de infit $(1,02)$ indica adequação das respostas dos participantes ao padrão esperado pelo modelo. No entanto, as estatísticas mínimo e máximo $(0,15$ e 4,41$)$ apontam pessoas que apresentam padrão inesperados de resposta quando níveis de traço latente estavam próximo ao nível de dificuldade do item. As mesmas características foram observadas quanto ao índice de ajuste outfit, ou seja, valor médio adequado $(1,02)$ e estatística mínimo e máximo $(0,15$ e 5,27$)$ que apontavam pessoas com padrões inesperados de resposta quando o nível de traço latente apresentado se localiza distante do nível de dificuldade do item, o que significa que pessoas com alto nível de traço latente deixavam de endossar itens fáceis, enquanto pessoas com baixos níveis de traço latente endossavam itens estimados como difíceis.

Por fim, buscou-se avaliar a possível presença de DIF nos itens do instrumento em função do sexo dos participantes. Os resultados são apresentados na Tabela 4 onde se verifica o nível de dificuldade dos itens para os homens, para as mulheres, o contraste entre os dois índices e os índices de probabilidade Mantel-Haenzel.

Conforme destacado no item método foram adotados como critérios para presença de DIF valores de contraste superiores a $0,42 \mathrm{e} / \mathrm{ou}$ probabilidade Mantel-Haenzel menor que 0,05 (Aguerri et al., 2007). Desta maneira, observa-se que apenas o item 3 "Se eu procurar ajuda, posso resolver a situação de violência" apresenta DIF, uma vez que ele é significativamente mais fácil de ser endossado pelas mulheres (dificuldade igual a -1,31) quando comparado aos homens (dificuldade igual a $-0,68$ ), resultados que 
TABELA 4

Função diferencial do item - DIF

\begin{tabular}{ccccc}
\hline Itens & Dificuldade Homens & Dificuldade Mulheres & DIF-Constrate & Probabilidade Mantel-Haenzel \\
\hline 1 & 0,19 & 0,24 & $-0,04$ & 0,878 \\
2 & $-0,03$ & $-0,30$ & 0,27 & 0,204 \\
3 & $-\mathbf{0 , 6 8}$ & $\mathbf{- 1 , 3 1}$ & $\mathbf{0 , 6 3}$ & $\mathbf{0 , 0 3 3}$ \\
4 & $-0,21$ & $-0,53$ & 0,32 & 0,147 \\
5 & 0,03 & 0,11 & $-0,08$ & 0,837 \\
6 & 0,28 & 0,62 & $-0,34$ & 0,390 \\
7 & 0,67 & 1,07 & $-0,41$ & 0,326 \\
8 & 0,05 & 0,29 & $-0,24$ & 0,502 \\
9 & 0,75 & 0,39 & 0,36 & 0,105 \\
10 & $-0,24$ & $-0,20$ & $-0,04$ & 0,275 \\
11 & $-0,77$ & $-0,82$ & 0,06 & 0,338 \\
12 & $-0,37$ & $-0,43$ & 0,06 & 0,707 \\
13 & $-0,12$ & 0,16 & $-0,28$ & 0,105 \\
14 & 0,25 & 0,50 & $-0,25$ & 0,359 \\
15 & 0,16 & 0,22 & $-0,06$ & 0,819 \\
\hline
\end{tabular}

denunciam que a diferença nos escores entre homens mulheres neste item pode ser influenciada por outros viesses, diferentes do construto latente. Este resultado destaca uma discussão já presente nos estudos de apoio social acerca das diferenças de percepção e busca de apoio social por parte de homens e mulheres. O sexo feminino tende a relatar maior percepção e uso de apoio social em situações cotidianas e/ou estressantes em detrimento do sexo masculino (Zanini, VerollaMoura, \& Queiroz, 2009).

\section{Considerações finais}

O presente estudo teve como objetivo a elaboração e o levantamento das primeiras evidências de validade de uma escala de autoeficácia para situações de vitimização. Os estudos relacionados ao conceito de autoeficácia e as formas como esta influencia ou se relaciona com os comportamentos humanos ou a saúde são crescentes na literatura internacional em Psicologia. No Brasil, também se observa um interesse sobre o construto, embora ainda sejam poucos os estudos que envolvam a elaboração de instrumentos de medida.

$\mathrm{O}$ desenvolvimento deste instrumento iniciou-se por meio de discussões teóricas e de revisão da bibliografia, com posterior elaboração de um conjunto de 17 itens sobre a autoeficácia para situações de vitimização. A análise de validade conceitual realizada por cinco juízes independentes manteve os 17 itens. Contudo, a análise dos dados da escala indicou a inadequação de dois itens (16 e 17) que obtiveram cargas fatoriais inferiores a 0,32. Estes itens foram suprimidos da escala. Ambos fazem referência a reações emocionais relacionadas a situação de violência (16 - "Fico nervoso em situações de violência"; 17 - "Perco o controle quando enfrento situações de violência") e talvez por isso seus índices de correlação com os demais itens não tenham sido satisfatórios. Entretanto trata-se de uma fonte de informação de eficácia a partir das experiências pessoais segundo Bandura (1997; 1999). Desta forma, a supressão destes itens da escala de eficácia geral atende aos critérios de adequação estatística, mas elimina a possibilidade de avaliação 1) das reações emocionais percebidas pelo indivíduo relacionadas a situação de violência; 2) de uma fonte de informação que auxilie na construção da crença de autoeficácia baseada em experiências pessoais. Estudos futuros que queiram avaliar estes conteúdos devem cuidar em associar medidas sensíveis a estes aspectos.

A solução fatorial retida em um único fator relacionado à crença de eficácia geral para situações de vitimização demostrou bom índice de precisão. Além disso, a TRI possibilitou a verificação das propriedades dos itens e dos participantes da pesquisa, demonstrando a capacidade da EAREV-15 de identificar pessoas com diferentes níveis de autoeficácia em situações de vitimização e, portanto, o potencial de utilidade do instrumento por profissionais práticos na construção de psicodiagnósticos ao revelar um importante atributo dos sujeitos avaliados. Mais especificamente, o presente estudo possibilita identificar diferentes níveis de crenças de autoeficácia aplicada a situações de violência o que permite, em pesquisas futuras, a comparação 
dos resultados de diferentes grupos segundo suas pontuações nesta escala e sua relação com a vivência de violência. Outros aspectos que merecem atenção em estudos futuros dizem respeito à obtenção de outras evidências de validade, como a relação com variáveis externas teoricamente relacionadas com este construto e o desenvolvimento de normas interpretativas para os escores brutos da EAREV-15. Para este último sugerese o emprego do procedimento Mapa de itens-pessoas, com base no MCP, para o desenvolvimento de normas baseadas nos itens da escala, conforme sugerido por
Primi, Wechsler, Nakano, Oakland e Guzzo (2014). Como limitação da EAREV-15 destaca-se o item 3, que apresentou grau de dificuldade diferente para homens e mulheres, influenciado por questões culturais e não apenas pelo construto latente avaliado. Sugerese, ainda, a necessidade de novos estudos que avaliem o resultado final da escala e sua aplicabilidade em outras amostras de adultos, em outros contextos que não estudantes universitários, haja vista que a pesquisa contou com uma mostra não aleatória e restrita a região centro-oeste do país.

\section{Referências}

Aguerri, M. E., Galibert, M. S., Lozzia, G. S., Abal, F. J. P., \& Attorresi, H. F. (2007). Medida del funcionamento diferencial del item en el marco de la teoria de respuesta al item. Interdisciplinaria, 24(1), 95-110.

Amantes, A. \& Coelho, G. (2015). Modelos politômicos. In H. F. Golino, C. M. A., Gomes, A., Amantes, \& G. Coelho (Eds.), Psicometria contemporânea: compreendendo os Modelos Rasch (pp. 155-183). São Paulo: Casa do Psicólogo

Ambiel, R. A. M. \& Noronha, A. P. P. (2011). Construção dos itens da Escala de Autoeficácia para Escolha Profissional. Psico-USF, 16(1), 23-32. http://dx.doi.org/10.1590/S1413-82712011000100004

Azevedo, L. R. (2013). Dialogues on the notion of victim and identity construction. Interface (Botucatu), 17(46), 515-522. http://dx.doi.org/10.1590/S1414-32832013000300002

Bandura, A. (1997). Self-Efficacy: The exercise of control. New York: W. H. Freeman Company. Retirado de: <http:// www.des.emory.edu/mfp/effbook1.html>.

Bandura, A. (1999). A social cognitive theory of personality. In L Pervin \& O. John (Eds.), Handbook of personality (pp. 154-196). New York: Guilford Publications. Retirado de: <http://des.emory.edu/mfp/Bandura1999HP.pdf>.

Bandura, A. (2006). Guide for constructing self-efficacy scales. In F. Pajares \& T. C. Urdan (Eds.), Self-efficacy beliefs of adolescentes (pp. 307-377). USA: Information Age Publishing. Retirado de: $<$ http://books.google.com.br/books $>$.

Brasil. Ministério da Saúde - Secretaria de Atenção à Saúde. Departamento de Atenção Básica. (2009). Por uma cultura de paz, a promoção da saúde e prevenção da violência. 44 p. Série F. Comunicação e Educação em Saúde.

Buja, A. \& Eyuboglu, N. (1992). Remarks on parallel analysis. Multivariate Behavioral Research, 27(4), 509-540. http:// dx.doi.org/10.1207/s15327906mbr2704_2

Dahlberg, L. L. \& Krug, E. G. (2007). Violência: um problema global de saúde pública. Ciência \& Saúde Coletiva, 11, 1163-1178. http://dx.doi.org/10.1590/S1413-81232006000500007

Finkelhor, D. (2007). Developmental victimology: the comprehensive study of childhood victimizations. In R. C. Davis, A. J. Luirigio \& S. Herman (Eds.), Victims of crime (3rd ed., pp. 9-34). Thousand Oaks, CA: Sage Publications.

Finotelli Junior, I. \& Capitão, C. G. (2011). Evidências de validade da versão brasileira da Escala de Autoeficácia Sexual - Função Erétil. Psico-USF, 1, 45-55. http://dx.doi.org/10.1590/S1413-82712011000100006

Linacre J. M. (2015). A user `s guide to Winsteps Ministep: Rasch-model computer programs. Retirado de: <http://www. winsteps.com>.

Lorenzo-Seva, U., \& Ferrando, P. J. (2006). FACTOR: A computer program to fit the exploratory factor analysis model. Behavioral Research Methods, Instruments and Computers, 38(1), 88-91. http://dx.doi.org/10.3758/BF03192753

Lorenzo-Seva, U., Timmerman, M. E., \& Kiers, H. A. L. (2011). The hull method for selecting the number of common factors. Multivariate Behavioral Research, 46, 340-364. http://dx.doi.org/10.1080/00273171.2011.564527

Mardia, K. V. (1970). Measures of multivariate skewnees and kurtosis with applications. Biometrika, 57(3), 519-530. http://dx.doi.org/10.1093/biomet/57.3.519

Martins, C. R. (2011). Abuso sexual e resiliência: Enfrentando as adversidades. Mal-estar e Subjetividade, 11(2), 727-750

Mognon, J. F. \& Santos, A. A. A. (2016). Escala de Autoeficácia para dirigir: Construção e avaliação preliminar das propriedades psicométricas. Estudos em Psicologia, 33(1), 127-136. http://dx.doi.org/10.1590/1982027520160001000013

Oliveira, M. C., Silva, B. R., Garcia, R. G., Melo-Silva, L. L., \& Teixeira, M. A. P. (2014). Escala de autoeficácia no aconselhamento de carreira: Adaptação e evidências de validade. Avaliação Psicológica, 13(3), 371-381.

Pajares, F. (2004). Overview of social cognitive theory and of self-efficacy. Retirado de: <www.emory.edu/EDUCATION/ $\mathrm{mfp} / \mathrm{eff} . \mathrm{html}$. 
Pajares, F. (2005). Current directions in self-efficacy research. Advances in motivation and achievement, 10, 1-49. Retirado de: <http://des.emory.edu/mfp/effchapter.html>.

Primi, R., Wechsler, S. M., Nakano, T., Oakland, T., \& Guzzo, R. S. L. (2014). Using item response theory methods with the Brazilian temperament scale for students. Journal of Psychoeducational Assessment, 32, 651-662. http://dx.doi. org/10.1177/0734282914528613

Polidoro, S. \& Casanova, D. C. G. (2015). Escala de autoeficácia acadêmica para o ensino médio: Busca de evidências psicométricas. Estudos Interdisciplinares em Psicologia, 6(1), 36-53.

Tabachnick, B. G. \& Fidell, L. S. (2012). Using multivariate statistics (6믈. ed. Boston: Allyn and Bacon.

Timmerman, M. E. \& Lorenzo-Seva, U. (2011). Dimensionality Assessment of Ordered Polytomous Items with Parallel Analysis. Psychological Methods, 16, 209-220. http://dx.doi.org/10.1037/a0023353

Waiselfisz, J. J. (2014). Mapa da violência 2014: Os jovens do Brasil (1ํㅡㄹ. ed.). Rio de Janeiro: FLACSO Brasil.

Williams, L. C. A., D’Affonseca, S. M., Correia, T. A., \& Albuquerque, P. P. (2011). Efeitos a longo prazo da vitimização escolar. Gerais: Revista Interinstitucional de Psicologia, 4, 187-199.

Wright, B. D. \& Masters, G. N. (1982). Rating scale analysis. Chicago: MESA.

Zanini, D. S., Verolla-Moura, A., \& Queiroz, I. P. A. R. (2009). Apoio social: Aspectos da validade de constructo em estudantes universitários. Psicologia em Estudo, 14(1), 195-202. http://dx.doi.org/10.1590/S1413-73722009000100023

Zimmerman, B. J. (2000). Self-efficacy: An essential motive to learn. Contempory Educational Psychology, 25, 82-89. http://dx.doi.org/10.1006/ceps.1999.1016

\author{
Autores: \\ Daniela Cristina Campos - Doutora, Pontifícia Universidade Católica de Goiás. \\ Margareth Regina Gomes Veríssimo de Faria - Doutora, Pontifícia Universidade Católica de Goiás. \\ Daniela Sacramento Zanini - Doutora, Pontifícia Universidade Católica de Goiás. \\ Evandro Morais Peixoto - Doutor, Pontifícia Universidade Católica de Campinas. \\ Endereço para correspondência: \\ Daniela S. Zanini \\ Rua 9 , no 769 \\ 74110-100 Goiânia, GO, Brasil \\ <dazanini@yahoo.com> \\ Recebido em: 15.02.2016 \\ Aceito em: 22.06.2016
}

